\title{
POLYGONS CIRCUMSCRIBED ABOUT CLOSED CONVEX CURVES
}

\author{
BY \\ MAHLON M. DAY
}

1. Introduction. This note gives two entirely different methods of proof of the following theorem and of certain of its extensions.

THEOREM 1. Given a symmetric closed convex curve $C$ in the plane, there exists a parallelogram $P$ circumscribed about $C$ so that the midpoint of each side of $P$ is on $C$.

Both proofs allow extension to more than four sides. The first proof, by means of the Poincare ring theorem [1] (1), is restricted to the plane but allows somewhat more general subdivision of the sides than simple bisection. The second proof, by minimal area, extends the theorem to circumscribed polyhedra of any number of sides in any number of dimensions.

The need for Theorem 1 first appeared in the course of some proofs of properties of unit spheres in normed linear spaces [2]. The applications to the problem which originally suggested Theorem 1 are clearer if, in a normed linear space, $x$ normal to $y$ is defined to mean $\|a x+y\| \geqq\|y\|$ for all real $a$; that is, that the line through $y$ parallel to $O x$ comes no closer to the origin than $y$. This relationship in a Euclidean space is equivalent to orthogonality of $x$ and $y$; in other normed spaces it retains some of the properties of orthogonality but is, in general, not symmetric $[2, \S 6]$. Theorem 1 asserts that in any twodimensional normed space there exist two points of norm one each normal to the other. The generalization, Theorem 4.1 , to $n$ dimensions asserts that in each n-dimensional normed linear space there exist $n$ points $x_{i}$ such that $\sum_{j \neq i} a_{j} x_{j}$ is normal to $x_{i}$ for any choice of $i=1, \cdots, n$ and real numbers $a_{j}$. Translating this notion of normality in the obvious way to Finsler spaces gives: Through every point $P$ of an n-dimensional locally-Minkowskian Finsler space there exist $n$ hypersurfaces each normal at $P$ to the intersection of all the others.

2. Proof by the ring theorem. Throughout this section assume that $C$ is a closed convex (not necessarily symmetric) curve with no flat sides; that is, that no line segment of positive length lies on $C$. (The general case of Theorem 1 is easily proved by approximation from this special case.) Choose a positive direction, say counterclockwise, around $C$. Then for each point $b$ outside or on $C$ there is a unique tangent line from $b$ to $C$ which passes in the chosen

Presented to the Society, April 28, 1944, and September 15, 1945; received by the editors September 27, 1946.

(1) Numbers in brackets refer to the bibliography at the end of the paper 
positive direction through a unique point $b^{\prime}$ on $C$. For any positive number $\lambda$ define a transformation $T_{\lambda}$ of the exterior of $C$ into itself by letting $T_{\lambda} b$ be the point on the given tangent from $b$ extended past $b^{\prime}$ so that the ratio of the distance from $b^{\prime}$ to $T_{\lambda} b$ to the distance from $b$ to $b^{\prime}$ is $\lambda$. Then, when $C$ is symmetric, a point $b$ is a corner of a parallelogram satisfying Theorem 1 if and only if $T_{1}^{2} b=-b$. To readjust this to a fixed point theorem let $S$ be the rotation of the exterior of $C$ into itself through $\pi$ radians in the negative direction. Then when $C$ is symmetric $b$ is a corner of a parallelogram satisfying Theorem 1 if and only if $S T_{1}^{2} b=b$. Theorem 1 is, therefore, a consequence of a far more inclusive result.

Lemma 2.1. If $\lambda_{i}, i=1, \cdots, n$, are positive numbers such that $\lambda_{1} \lambda_{2} \cdots \lambda_{n}$ $=1$, each of the transformations $S T_{\lambda_{1}} T_{\lambda_{2}} \cdots T_{\lambda_{n}}$ (for $n \geqq 2$ ) and $S^{2} T_{\lambda_{1}} T_{\lambda_{2}}$ $\cdots T_{\lambda_{n}}$ (for $\left.n \geqq 3\right)$ has a fixed point exterior to $C$.

We shall prove this by means of the Poincare ring theorem which is stated in G. D. Birkhoff's book [1, p. 165] as follows: Given a ring $0<a \leqq \rho \leqq b$ in the $\rho, \theta$ plane $(\rho, \theta$ being polar coordinates) and a one-to-one, continuous, area-preserving transformation $T$ of the ring into itself which advances points on $\rho=a$ and regresses points on $\rho=b$, then there will exist at least two points of the ring invariant under $T$.

The proof given can easily be shown to hold as well for the unbounded ring exterior to $C$, completed with an ideal circle at infinity. Since $\left\|T_{\lambda} b+\lambda b\right\|$, the distance from $T_{\lambda} b$ to $-\lambda b$, is precisely $1+\lambda, T_{\lambda}$, as well as $S$, can be defined continuously on this extended ring, so the transformations mentioned in the lemma are homeomorphisms of this extended ring. For every $\lambda, T_{\lambda} b=b$ for $b$ on $C$, so $S T_{\lambda_{1}} \cdots T_{\lambda_{n}}$ turns points on $C$ through $-\pi$ radians. Each $T_{\lambda}$ turns points on the circle at infinity through $+\pi$ radians; hence, for $n \geqq 2$, $S T_{\lambda_{1}} \cdots T_{\lambda_{n}}$ turns points on the circle at infinity through $(n-1) \pi>0$ radians. Similarly, for $n \geqq 3, S^{2} T_{\lambda_{1}} \cdots T_{\lambda_{n}}$ turns points on the circle at infinity through $(n-2) \pi$ radians. Hence the ring theorem will prove 2.1 if it can be shown that the given transformations are area-preserving. This is a simple consequence of the following lemma.

LEMMa 2.2. $T$ distorts areas in the ratio $\lambda^{2}: 1$.

Let $(p, \mu)$ be "polar" cocrdinates for a point $b$ exterior to $C$, defined so that $p$ is the distance along the tangent from $b$ to $C, \mu$ is the inclination of that tangent. It is easily shown that the area of the figure $F$ bounded by curves $\mu=\mu_{1}, \mu=\mu_{2}, p=p_{1}$, and $p=p_{2}$, with $0 \leqq p_{1} \leqq p_{2}<\infty$ and $0 \leqq \mu_{2}-\mu_{1} \leqq 2 \pi$, is $\left(p_{2}^{2}-p_{1}^{2}\right)\left(\mu_{2}-\mu_{1}\right) / 2$. (This is a very familiar formula if $C$ is shrunk to a point.) A similar computation with $T_{\lambda} F$ shows that its area is $\lambda^{2}$ times as great; since every open set can be approximated by sums of such elementary figures, $T_{\lambda}$ multiplies all areas by $\lambda^{2}$.

From this lemma and the assumption $\lambda_{1} \cdots \lambda_{n}=1$ it follows that 
$T_{\lambda_{1}} \cdots T_{\lambda_{n}}$ preserves areas. Since $S$ obviously preserves areas, Lemma 2.1 follows from the ring theorem. Translated back to the discussion of polygons the two conclusions of 2.1 give the following geometric results:

THEOREM 2.3. If $C$ is a closed convex curve with no flat sides and if $\lambda_{1} \cdots \lambda_{n}$ $=1, n \geqq 3$, then there exists an $n$-sided convex polygon $P$ circumscribed about $C$ such that, counting from a properly chosen vertex, the point $x_{i}$ which divides the ith side in the ratio $\lambda_{i}: 1$ is on $C$ for $i=1, \cdots, n$. If, moreover, $C$ is symmetric and $n \geqq 2$, then a symmetric $2 n$-sided polygon with the same property can be circumscribed about $C$.

Part of Theorem 1 is the symmetric case $n=2, \lambda_{1}=\lambda_{2}=1$. To remove the restriction that $C$ have no flat sides, approximate a general symmetric closed convex $C$ by a sequence $\left\{C_{r}\right\}$ with no flat sides. The parallelograms $P_{n}$ attached to the curves $C_{n}$ have a convergent subsequence whose limit fits in the proper way around $C$. The only question which might arise here is of the existence of a convergent subsequence. It is easily shown that if $T_{\lambda} b=k b$ for any negative $k$, then $\|b\|<1+2 / \lambda$; hence if $b$ is any corner of any $P_{n}$, it lies within a dilation of $C_{1}$ to three times its original size. Compactness can then be used to pick a convergent subsequence of the corners of the given sequence $\left\{P_{n}\right\}$.

3. A special proof of Theorem 1. Using most of the steps of the proof of the preceding section, a proof of Theorem 1 can be given in which the ring theorem is avoided by finding a fixed point of $S T_{1}^{2}$ directly. Begin by supposing that $C$ has neither flat sides nor corners. Let $\phi(b)$ be the angle measured in the positive direction between the half-ray from 0 to $b$ and that from 0 to $T^{2} b$. The first step is to show that the equation $\phi(b)=\pi$ has exactly one solution $b(\theta)$ on the half-ray in the direction $\theta$ from 0 and that $\|b(\theta)\|<3$. Define $C_{0}=\{b(\theta) \mid 0 \leqq \theta<2 \pi\}=\{b \mid \phi(b)=\pi\} ; C_{0}$ is closed and bounded; a compactness argument proves that $b(\theta)$ is continuous. It quickly follows that $C_{0}$ is a simple closed symmetric curve.

By Lemma 2.2, $T_{1}^{2}$ is area preserving. Hence $T_{1}^{2} C_{0}$ must intersect $C_{0}$ at some point $b_{1}$; that is, there is a point $b_{2}$ of $C_{0}$ such that $T_{1}^{2} b_{2}=b_{1}$; by symmetry of $C_{0}$ and the fact that $C_{0}$ cuts each half-ray only once, it follows that $b_{1}=T_{1}^{2} b_{2}=-b_{2}$. This means that $b_{2}, T_{1} b_{2}, T_{1}^{2} b_{2}$, and $T_{1}^{3} b_{2}$ are the corners of a parallelogram satisfying Theorem 1 . Theorem 1 can now be proved by the same approximation argument as before.

4. The minimal method. Theorem 1 is an immediate consequence of two facts: Any such $C$ has circumscribed about it a parallelogram of minimal area, and any minimal $P$ about $C$ has the desired property. This can be proved very simply in $n$ dimensions as well.

THEOREM 4.1. If $C^{n}$ is a symmetric, closed, convex body in the Euclidean $n$ space $E^{n}, n \geqq 2$, there exists an $n$-parallelopiped $P^{n}$ circumscribed about $C^{n}$ in such a way that the midpoint of each face of $P^{n}$ is on $C^{n}$. 
There is an $n$-parallelepiped $P^{n}$ about $C^{n}$ with minimal volume; it has the desired property. If $L_{i}, i= \pm 1, \pm 2, \cdots, \pm n$, are the $(n-1)$-dimensional hyperplanes containing the faces, $S_{ \pm i}$, of $P^{n}$, and if $L_{-i}$ is parallel to $L_{i}$, suppose that the midpoint of the face $S_{n}$ is not on $C^{n}$. Let $L_{i}^{\prime}, i=1, \cdots, n-1$, be parallel to $L_{i}$ and pass through the origin; then the intersection of all $L_{i}^{\prime}$ is a line through the origin. Let $p$ and $-p$ be its points of intersection with $C^{n}$. Draw parallel hyperplanes of support to $C^{n}$ through $p$ and $-p$ enclosing a new parallelopiped $Q^{n}$; also draw the hyperplanes parallel to $L_{n}$ but passing through $p$ and $-p$ defining another parallelepiped $R^{n}$. Then $Q^{n}$ and $R^{n}$ have the same volume; since $L^{n}$ does not pass through $p$ or $-p$, the points $\pm p$, and hence all of $R^{n}$, lie inside $P^{n}$. Hence the volume of $Q^{n}$ is less than that of $P^{n}$.

Theorem 2.3 in the special case $\lambda_{1}=\lambda_{2}=\cdots=\lambda_{n}=1$ can also be proved by the minimal method which, for this special choice of the $\lambda_{i}$, adds the information that an $n$-sided polygon of the desired type exists if and only if $C$ is not itself a polygon of fewer than $n$ sides. This result can also be extended in part to $n$ dimensions by means of the following lemma.

Lemma 4.2. Let $C^{k}$ be a bounded convex body in the k-dimensional Euclidean space $E^{k}$ and let $P^{k}$ be a convex "polyhedron" of $n$ faces circumscribed about $C^{k}$. If $S$ is a face of $P^{k}$ such that the centroid $p$ of $S$ is not on $C^{k}$, then there is a convex polyhedron $\Pi^{k}$ circumscribed about $C^{k}$ with smaller volume than that of $P^{k}$.

Here "volume" and "area" are the ordinary Lebesgue measures of $k$ and $k-1$ dimensions. Let $p_{1}$ be the point of contact of $C^{k}$ and $S$ which is nearest to the centroid $p$ of $S$, let $L$ be the intersection of $S$ with the hyperplane of points equidistant from $p_{1}$ and $p$. Then $L$ does not touch $C$ or $p$ so $S$ can be rotated about $L$ through a small angle $\theta$ to a position $S(\theta)$ which does not touch $C^{k}$. Since the moment $M$ of $S$ about $L$ is not zero, for small $\theta$ the decrease in volume due to replacing $S$ by $S(\theta)$ is approximately $M \tan \theta$. Let $\Pi^{k}$ be circumscribed about $C^{k}$ and have a face parallel to $S(\theta)$ replacing $S$; then the volume of $\Pi^{k}$ is still smaller than that of the polyhedron with face $S(\theta)$. If $\theta$ is small enough $\Pi^{k}$ has the same number of faces as $P^{k}$.

From this lemma comes a great extension of Theorem 1 to polyhedra of many faces in higher dimensions.

THEOREM 4.3. Let $C^{k}$ be a symmetric closed convex body in the $k$-dimensional linear space $E$ and let $n$ be an integer not less than $k$. If $P$ is a symmetric convex polyhedron of $2 n$ sides circumscribed about $C^{k}$ with minimal volume, then the centroid of each face of $P^{k}$ is on $C^{k}$.

Deleting symmetry gives the usual analogues. As in the plane it is clearly sufficient for existence of a $P^{k}$ of $2 n$ sides with minimal volume that $C$ not be a polyhedron of less than $2 n$ sides. Hence this is a sufficient condition for existence of a $P^{k}$ with the centroid of each face of $P^{k}$ on $C^{k}$. However, the ex- 
ample of a regular octahedron around a cube shows that $C^{k}$ may have fewer sides than $P^{k}$ and still have the midpoint of each side of $P^{k}$ on $C^{k}$.

Added in proof. A theorem of a recent note of Taylor [3] gives an alternative proof of Theorem 4.1. He selects from all those parallelopipeds for which the centers of the faces are in or on $C$ one of maximal volume, and then shows that it is circumscribed about $C$. If $C$ is an octohedron, Taylor's proof gives for $P$ the cube whose face centers are at the corners of $C$, while the circumscribed parallelopipeds of minimal volume (there are many) have one-fourth the volume of the cube.

\section{BIBLIOGRAPHY}

1. G. D. Birkhoff, Dynamical systems, Amer. Math. Soc. Colloquium Publications, vol. 9.

2. M. M. Day, Some characterizations of inner-product spaces, Trans. Amer. Math. Soc. vol. 62 (1947) pp. 320-327.

3. A. E. Taylor, $A$ geometric theorem and its application to biorthogonal systems, Bull. Amer. Math. Soc. vol. 53 (1947) pp. 614-616.

UNIVERSITY OF ILLINOIS, URBANA, ILL. 